\title{
Microstructural Characterization of Polycrystalline Diamond Sintered at Ultrahigh Pressures
}

\author{
E.G. Minnaar ${ }^{1}$, J.E. Westraadt ${ }^{1}$ and J.H. Neethling ${ }^{1}$ \\ ${ }^{1 .}$ Centre for High Resolution Transmission Electron Microscopy, Nelson Mandela Metropolitan \\ University, Port Elizabeth, South Africa.
}

Polycrystalline diamond (PCD) is produced during a high-pressure high-temperature process (5.5 - 7.7 $\mathrm{GPa}$ and $\left.1350-1700{ }^{\circ} \mathrm{C}\right)$ from micron $(1-100 \mu \mathrm{m})$ sized diamond powders. This material has unique properties, such as high hardness, excellent thermal conductivity and high wear resistance [1]. It is therefore widely used in a variety of cutting, drilling and milling applications such as oil and gas drilling, non-ferrous materials machining and asphalt road planing. The diamond powder is placed on a substrate of WC/Co and consolidated in situ. The cobalt melts and infiltrates into the diamond structure during processing, causing the diamond grains to bond together.

Conventional PCD materials experience a decline in tool performance after exposure to $750{ }^{\circ} \mathrm{C}$ [2]. This effect has been attributed to the residual cobalt $(\sim 10$ vol. $\%)$ in the compact, which promotes the conversion of diamond to graphite at low pressures [3]. In addition, differences in the thermal expansion coefficients of diamond and Co induce internal residual stresses, which breaks the diamond-diamond bonds generating microcracks in the PCD at operating temperatures above $730{ }^{\circ} \mathrm{C}$. One possible solution would be to limit the cobalt content in the final sintered compact. Various approaches have been applied to achieve this, which include acid leaching [2], controlled binder additions and/or the use of elevated sintering pressures [4]. The resulting microstructure of this material is a function of the processing parameters (grain size, pressure, temperature) as well as the method used to introduce cobalt into the diamond powder. The objective of this study was to investigate the effect of different processing parameters on the PCD microstructure.

Diamond powders $(\sim 12 \mu \mathrm{m})$ were sintered at pressures ranging from 5.5-15 GPa and temperatures ranging from 1800-2300 ${ }^{\circ} \mathrm{C}$; (1) without cobalt added (hot compaction), (2) mixing 2 vol. \% cobalt with the diamond powder (admixed) and (3) via infiltration from a cobalt layer (infiltration). Raman spectroscopy was used to determine the ratio of diamond $\left(1332 \mathrm{~cm}^{-1}\right)$ to graphite $\left(1580 \mathrm{~cm}^{-1}\right)$ in the sintered compacts. SEM imaging using backscattered electrons ( $3 \mathrm{keV}$ ) was used to evaluate the amount of cobalt present in the infiltrated samples. Quantitative image analysis of the SEM images was performed using ImageJ. Focused ion beam SEM (FIB-SEM) was used to prepare thin sections for the TEM investigations. HAADF-STEM imaging with electron energy loss spectroscopy (EELS) was used to investigate the diamond-diamond and cobalt-diamond interfaces to evaluate the sintering quality.

Figure 1 shows an overview of the results of this study. The hot compact samples all contained significant amounts of graphite as measured by Raman spectroscopy; the relative graphite/diamond peak intensities were inversely proportional to the sintering pressure. STEM-EELS investigations (not shown) confirmed the presence of graphite between the diamond-diamond interfaces.

Raman spectroscopy and STEM-EELS investigations of the admixed (2 vol. \% Co) PCD showed that the sample sintered at $7.7 \mathrm{GPa}$ contained significant amounts of graphite. Low-pressure regions in the pores between the diamond grains converted to graphite in the presence of the admixed cobalt. The sintering of 
this cobalt admixed diamond powder at $15 \mathrm{GPa}$ and $2300{ }^{\circ} \mathrm{C}$ plastically deformed the diamond structure to such an extent that these low-pressure pores were eliminated. This resulted in a well-sintered compact without graphitization as measured by Raman spectroscopy.

The cobalt content of the infiltrated samples was a strong function of the sintering pressure. The diamond powder sintered at $5.5 \mathrm{GPa}$ contained $10.2( \pm 0.3)$ vol. \% cobalt compared to $4.5( \pm 1.1)$ vol. \% for the sample sintered at $15 \mathrm{GPa}$. This is probably due to the effect that the sintering pressure has on the open porosity just before cobalt infiltration. Higher pressures will plastically deform the diamond structure to a greater extent, thus leaving relatively less volume for cobalt infiltration. Atomic traces of cobalt were found between the diamond-diamond interfaces as shown in Figure 2d. STEM-EELS of all infiltrated samples detected minute levels of graphite surrounding isolated cobalt pools, most likely related to inhomogeneous pressure variations within the diamond compact.

In summary, we cobalt content in the final sintered compact is a strong function of the pressure. Although this could potentially improve the wear behaviour of the material, the cost implications of sintering PCD at elevated pressures might prove to be prohibitive.

References:

[1] Y Zhang et al, High Pressure Research 29 (2009), p. 325.

[2] F Bellin et al in "The Current state of PCD Technology", World Oil (October 2010) p. 53.

[3] D Meng et al, Journal of Superhard Materials 37 (2015), p. 67.

[4] M Akaishi et al, Journal of the American Ceramic Society 70 (1987), p. 237.

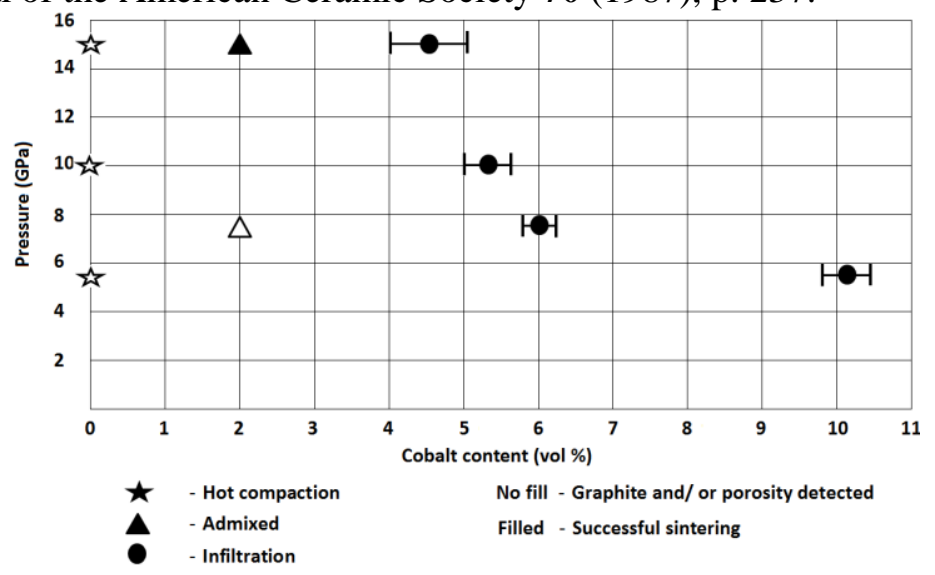

Figure 1. Summary of the sintering quality of the hot compact, admixed and infiltrated samples.

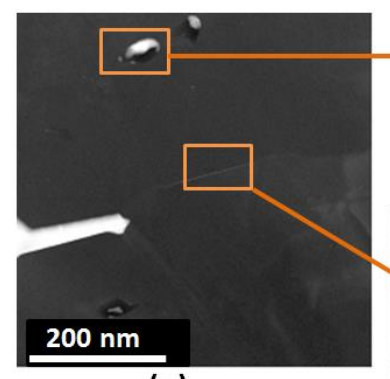

(a)

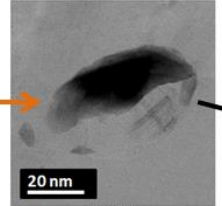

(b)

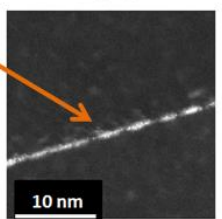

(d)

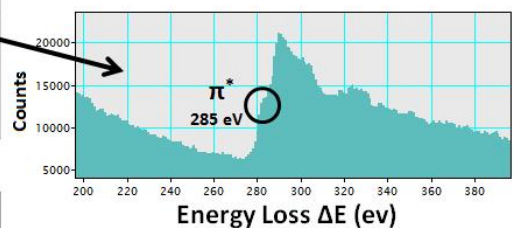

(c)

Figure 2. (a) HAADF-STEM image of the 5.5 GPa infiltrated PCD showing the diamond-diamond grain boundary, (b) HR-TEM image of cobalt inclusion with graphitic sheets, (c) Graphite EELS spectra, (d) HAADF-STEM image showing a diamond-diamond boundary separated by cobalt layer. 